\title{
Mononucleotide Marker Panel
}

National Cancer Institute

\section{Source}

National Cancer Institute. Mononucleotide Marker Panel. NCI Thesaurus. Code C96288.

A set of genetic loci where each locus contains a mononucleotide (single base pair) repeat sequence. Changes in the length of the repeat indicate mutations in mismatch repair genes resulting in microsatellite instability. 